\title{
Development of a stimuli-responsive polymer nanocomposite toward biologically optimized, MEMS-based neural probes
}

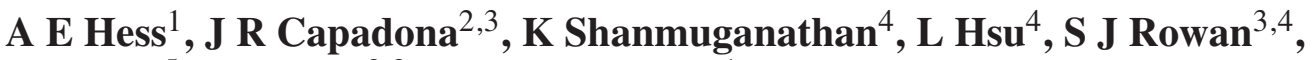 \\ C Weder ${ }^{5}$, D J Tyler ${ }^{2,3}$ and C A Zorman ${ }^{1}$ \\ ${ }^{1}$ Department of Electrical Engineering and Computer Science, Case Western Reserve University, \\ Cleveland, OH, USA \\ ${ }^{2}$ Rehabilitation R\&D, Louis Stokes Cleveland VA Medical Center, Cleveland, OH, USA \\ ${ }^{3}$ Department of Biomedical Engineering, Case Western Reserve University, Cleveland, OH, USA \\ ${ }^{4}$ Department of Macromolecular Science and Engineering, Case Western Reserve University, Cleveland, \\ $\mathrm{OH}$, USA \\ ${ }^{5}$ Adolphe Merkle Institute and Fribourg Center for Nanomaterials, University of Fribourg, Switzerland \\ E-mail: allison.hess@case.edu
}

\begin{abstract}
This paper reports the development of micromachining processes and mechanical evaluation of a stimuli-responsive, mechanically dynamic polymer nanocomposite for biomedical microsystems. This nanocomposite consists of a cellulose nanofiber network encased in a polyvinyl acetate matrix. Micromachined tensile testing structures fabricated from the nanocomposite displayed a reversible and switchable stiffness comparable to bulk samples, with a Young's modulus of $3420 \mathrm{MPa}$ when dry, reducing to $20 \mathrm{MPa}$ when wet, and a stiff-to-flexible transition time of $\sim 300 \mathrm{~s}$. This mechanically dynamic behavior is particularly attractive for the development of adaptive intracortical probes that are sufficiently stiff to insert into the brain without buckling, but become highly compliant upon insertion. Along these lines, a micromachined neural probe incorporating parylene insulating/moisture barrier layers and $\mathrm{Ti} / \mathrm{Au}$ electrodes was fabricated from the nanocomposite using a fabrication process designed specifically for this chemical- and temperature-sensitive material. It was found that the parylene layers only slightly increased the stiffness of the probe in the wet state in spite of its much higher Young's modulus. Furthermore, the Ti/Au electrodes exhibited impedance comparable to Au electrodes on conventional substrates. Swelling of the nanocomposite was highly anisotropic favoring the thickness dimension by a factor of 8 to 12 , leading to excellent adhesion between the nanocomposite and parylene layers and no discernable deformation of the probes when deployed in deionized water.
\end{abstract}

\section{Introduction}

Advances in process technologies for polymers such as poly(pxylylene) (also known as parylene), poly(dimethylsiloxane) (PDMS), polyimide and the epoxy-based photoresist SU-8 have enabled the development of biomedical microsystems that could otherwise not be realized if silicon were used as the main structural material. The chemical, electrical and mechanical properties of these polymers can often be 'tuned' during synthesis but cannot be reversibly altered once the 
polymer is formed. Recent progress in polymer development has been toward stimuli-responsive materials that allow for in situ variation of polymer properties. Applications for such devices include controlled, or even targeted, drug delivery throughout the body using $\mathrm{pH}$-sensitive polymers with variable volume [1], and microfluidic valves using electrically triggered hydrogels [2].

For mechanically dynamic, stimuli-responsive polymers with potential utility in microelectromechanical systems (MEMS), biological systems offer a rich source of inspiration. For example, the sea cucumber (Cucumaria frondosa) dermis displays a switchable stiffness [3], exhibiting a storage modulus change from $\sim 5 \mathrm{MPa}$ under normal conditions to $\sim 50 \mathrm{MPa}$ under perceived threat [4]. The nanocomposite structure of the dermis is comprised of stiff collagen fibers dispersed throughout a soft fibrillin matrix [3, 5, 6], with stresstransfer interactions between the collagen fibers modulating the stiffness.

Using the sea cucumber dermis behavior and mechanism as a model, we have recently developed a polymer nanocomposite with switchable stiffness [7, 8]. Stiff, highaspect-ratio cellulose nanofibers or whiskers $(E=143 \mathrm{GPa}$ [9]) obtained from tunicates, filter-feeding sea creatures, serve as the stiff, interlocking nanofiber filler within a highly flexible poly(vinyl acetate) (PVAc) matrix. Noncovalent hydrogen bond interactions between hydroxyl surface groups on the cellulose nanofibers, also referred to as tunicate whiskers (TW), control stress-transfer interactions throughout the PVAc nanocomposite (PVAc-TW). When the material is dehydrated, the hydrogen bonds form between the cellulose nanofibers, creating a reinforcing and stiffening network throughout the material. Upon immersion in hydrogen-bond forming liquids (i.e. water), the hydrogen bond interactions between nanofibers are displaced, and the stiffness is reduced by two to three orders of magnitude. In addition, water also plasticizes the PVAc matrix, reducing its glass transition temperature to well below body temperature, further enhancing the mechanical contrast between the stiff and soft states of the nanocomposite. Testing of razor-cut bulk samples that were nominally 2-3 mm wide, 25-30 mm long, and 100-300 $\mu \mathrm{m}$ thick by dynamic mechanical thermal analysis, has shown that the storage modulus of the nanocomposite ranges from $4.2 \mathrm{GPa}$ (for $15 \mathrm{w} / \mathrm{w} \%$ tunicate whiskers, TW) in the dry state at room temperature to about $12 \mathrm{MPa}$ in the wet state at $37{ }^{\circ} \mathrm{C}[7,10]$.

One application of this water-responsive, mechanically dynamic nanocomposite is as the principal structural material for intracortical neural recording probes [11]. These needlelike probes must be sufficiently stiff to penetrate cortical tissue through the pia mater without buckling [12, 13], but should also be highly flexible to resemble the mechanics of brain tissue once deployed [14, 15]. Silicon-based intracortical probes are easily inserted into the brain without buckling due to their high Young's modulus ( $\sim 160 \mathrm{GPa})$, but micromotion between the mechanically dissimilar Si probes and cortical tissue may significantly increase the risk of tissue damage, potentially triggering an immune response that can result in the formation of an insulating cellular sheath around the probe [16, 17]. As the sheath forms, the ability to record neuron activity is diminished and eventually lost, limiting electrode viability to only several months [18]. To alleviate problems related to mechanical mismatch, polymerbased cortical probes (i.e. from polyimide and parylene) have been developed [13, 19-21], reducing the probe stiffness by approximately two orders of magnitude compared to $\mathrm{Si}$. However, as Young's modulus of brain tissue is $\sim 10 \mathrm{kPa}$ [22-24], and the polymer-based probes have a modulus of $\sim 2.5 \mathrm{GPa}$, the mechanical mismatch is still considerable. Further, the overall stiffness of the conventional polymerbased probes is too low to penetrate the brain without buckling, unless stiff backbones [13] or gel-filled microfluidic channels are used [21]. The fixed mechanical properties of typical materials used in micromachined $\mathrm{Si}$, polyimide, or parylene neural probes cannot be optimized for both brain penetration and long-term deployment. Thus PVAc-TW, and the new class of mechanically adaptive polymer nanocomposites that it represents [8, 25, 26], provides a potential advantage over conventional polymers for penetrating probe-based neural interfacing in that the nanocomposite is sufficiently rigid for probe insertion, but upon deployment absorbs biological fluids, becoming much more pliable to better mechanically match brain tissue. Fabrication of neural probes from such polymers will enable researchers to evaluate the role that mechanics plays in long-term neural recording.

Three primary questions in the development of a mechanically dynamic intracortical probe from a PVAcTW substrate concern (1) whether the chemo-responsive switchable stiffness behavior demonstrated previously in bulk samples, with dimensions much larger than typical intracortical probes, scales to MEMS dimensions; (2) how to fabricate microscale, multi-material structures using PVAcTW substrates; and (3) whether the device fabrication methods affect the chemo-responsive PVAc-TW behavior. In a preliminary study investigating these questions, we reported that laser micromachining was an adequate method of producing micro-scale PVAc-TW structures that displayed dynamic mechanical behavior comparable to bulk samples [11]. While a method to pattern the PVAc-TW itself was described, these structures did not include metal or insulator features defined using photolithographic processes. This paper builds significantly upon our previous work by reporting on the development of micromachining techniques to produce a multilayered intracortical probe suitable for chronic cortical recordings. The mechanical properties of laser-patterned, MEMS-scale PVAc-TW test structures were assessed to determine how well the chemo-responsive behavior translates to structures subjected to micromachining techniques, as well as the response time of micromachined PVAc-TW structures upon exposure to water. Parylene $\mathrm{C}$ was evaluated for use as a moisture barrier between the moisture-absorbing PVAcTW and the electrically conducting Ti/Au features. The adhesion of thin film multilayers to the PVAc-TW under dry and wet conditions, as well as the electrode impedance of a micromachined probe, was assessed to determine the aptitude for long-term deployment of these structures. 
Table 1. Laser-cutting parameters for patterning PVAc-TW.

\begin{tabular}{|c|c|}
\hline Parameter & Setting \\
\hline Power & $0.5 \mathrm{~W}$ \\
\hline Speed & $56 \mathrm{~mm} \mathrm{~s}^{-1}$ \\
\hline Resolution & 1000 pulses per inch \\
\hline Number of passes & 1 \\
\hline
\end{tabular}

\section{Nanocomposite micromachining}

\subsection{Solution casting of the PVAc-TW nanocomposite}

The two-component PVAc-TW nanocomposite was prepared using a solution-casting technique, as previously described [8]. Briefly, cellulose nanofibers were isolated from sea tunicates by acid hydrolysis in sulfuric acid. The whiskers were then purified and redispersed in dimethylformamide (DMF), while PVAc pellets were separately dissolved in DMF. The whisker and PVAc solutions were combined and the mixture was cast into a Teflon dish. The solvent was removed by heating at $60{ }^{\circ} \mathrm{C}$ in a vacuum oven. Finally, the composite was compressed to form planar specimens 50 to $150 \mu \mathrm{m}$ in thickness, with a cellulose nanofiber content of $15 \%$ by volume.

\subsection{Laser micromachining}

PVAc-TW is difficult to process because it degrades upon exposure to most acids, bases, and organic solvents used in microfabrication, as well as to temperatures exceeding $\sim 120{ }^{\circ} \mathrm{C}$. Further, because PVAc is moisture-absorbent, wet chemicals must be avoided during processing to prevent implanted biomedical microdevices from leaching any absorbed chemicals. Laser micromachining is a dry process with only transient, localized increases in temperature, and thus was chosen as the patterning method for PVAc-TW microstructures.

PVAc-TW freestanding films were prepared for laser patterning by pressing the film to a Si wafer with mild pressure while applying heat from a hotplate at $70{ }^{\circ} \mathrm{C}$ for $3 \mathrm{~min}$, allowing the sheet to conform to the flat surface. A direct-write $\mathrm{CO}_{2}$ laser (VersaLaser), with tunable laser power, speed, and resolution, was then focused onto the PVAc-TW surface, and the material was cut using high power density focal optics to achieve a spot size of $25 \mu \mathrm{m}$. Optimal laser-cutting parameters for minimizing feature size and maximizing repeatability and uniformity are listed in table 1. After micromachining, the microstructures were carefully removed from the wafer using tweezers. An example of a laser-micromachined PVAc-TW microstructure is shown in the SEM image in figure 1.

\subsection{Integrating metal electrodes on the PVAc-TW substrate}

While $\mathrm{CO}_{2}$ laser micromachining can be employed to pattern the polymer nanocomposite, it cannot be used to pattern metal electrodes and interconnects. Further, a moistureresistant insulating layer was required to prevent moisture from corroding the metal features or degrading signal isolation. Thus, the laser-cut PVAc-TW microstructures were coated

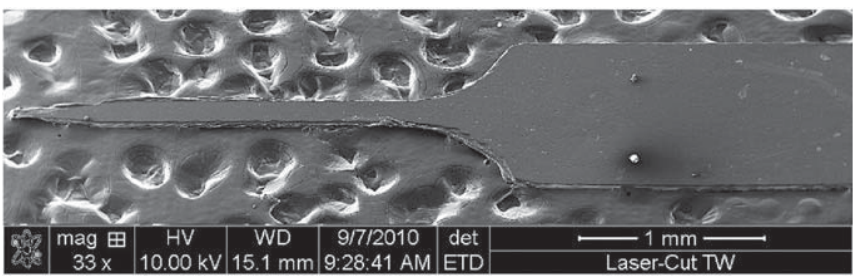

Figure 1. Scanning electron micrograph of a laser-micromachined cortical probe with a shank width of $180 \mu \mathrm{m}$.

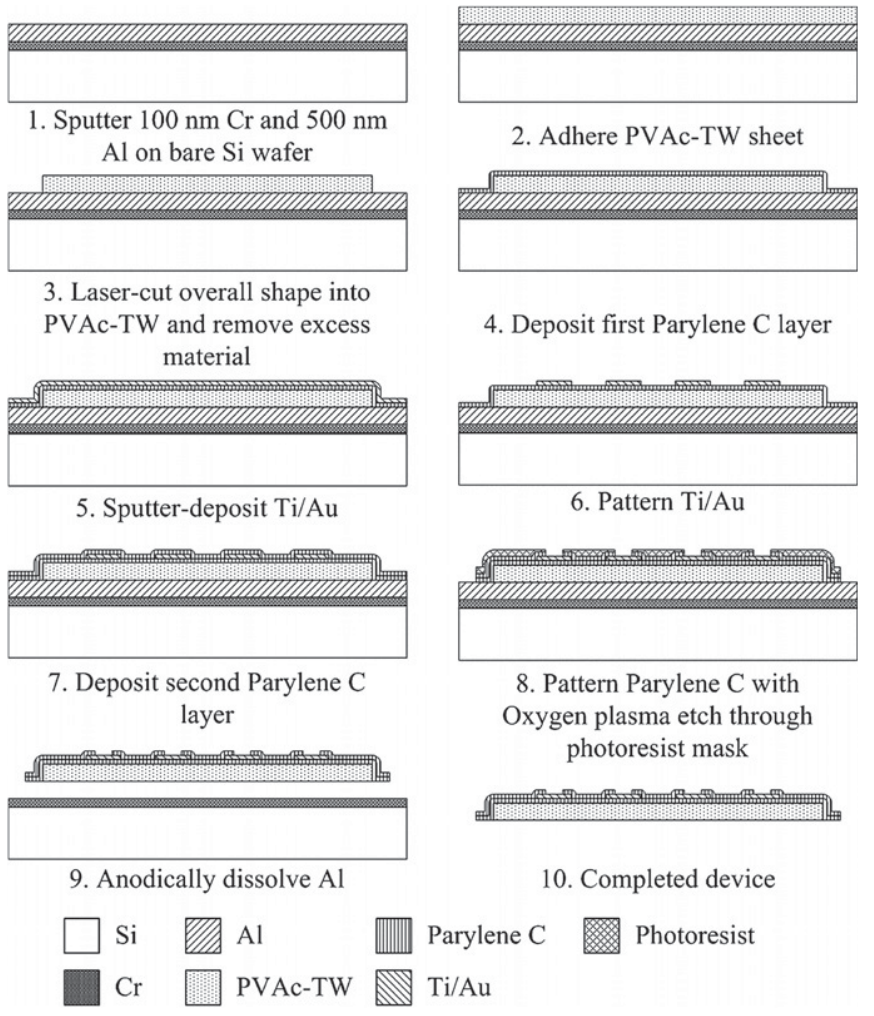

Figure 2. Fabrication process for integrating metal electrodes onto a chemically sensitive PVAc-TW substrate.

with a $1 \mu \mathrm{m}$ thick Parylene $\mathrm{C}$ insulating/moisture barrier layer. Parylene was chosen because it is biocompatible, highly moisture resistant, can be applied as uniform coating by vapor deposition in a wide range of thicknesses, and is deposited near room temperature. While parylene was primarily applied to protect the metal features from the moisture absorbed by PVAc-TW, it also served to protect the PVAc-TW from the wet chemicals used to pattern the metal features on the nanocomposite substrate. The full process is detailed below, and illustrated schematically in figure 2 .

The substrate was prepared by first sputter-depositing a sacrificial $\mathrm{Cr} / \mathrm{Al}(100 \mathrm{~nm} / 500 \mathrm{~nm})$ film on a bare $\mathrm{Si}$ wafer, which served as a mechanical substrate for handling throughout the microfabrication processes. The PVAc-TW freestanding film, ranging in thickness from 50 to $100 \mu \mathrm{m}$, was then adhered to the wafer by applying mild pressure to the film on the wafer while heating on a hotplate at $70{ }^{\circ} \mathrm{C}$ for $3 \mathrm{~min}$. The overall device geometry was then laser-cut into the PVAc-TW using the parameters in table 1. Excess PVAcTW in the field region was carefully removed with tweezers, leaving the PVAc-TW microstructures adhered to the wafer. 


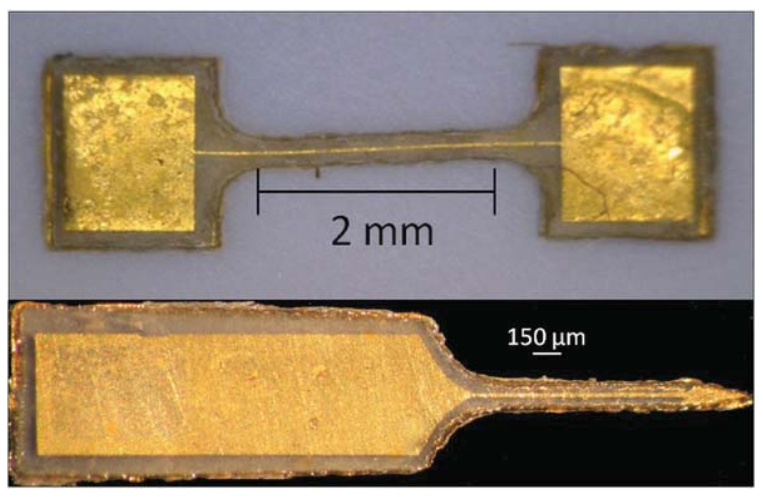

Figure 3. Top: micromachined dogbone structure with lithographically defined Ti/Au pads and trace; bottom: a laser-micromachined PVAc-TW cortical probe with a lithographically defined Ti/Au electrode, interconnects, and a parylene capping layer.

The first $1 \mu \mathrm{m}$ thick parylene film was vapor-deposited, coating the top surface and sidewalls of the PVAc-TW microstructures, as well as the exposed areas of the wafer. This parylene film served the dual purposes of protecting the PVAc-TW from subsequent chemical processing, and providing a moisture barrier to insulate the metal features from the moisture absorbed by PVAc-TW. The parylene coating was intentionally kept thin, because with a Young's modulus of $2.75 \mathrm{GPa}$ [27], it could easily dominate the mechanical properties of the structure. Next, a $50 \mathrm{~nm}$ thick Ti adhesion layer and a $200 \mathrm{~nm}$ thick Au layer were sputter-deposited, and subsequently patterned using a Shipley 1813 photoresist mask and Au etchant and a buffered oxide etchant dip. The photoresist was then removed with acetone, and the wafer was rinsed with isopropanol.

To electrically insulate the patterned metal structures from the biological environment, a $1 \mu \mathrm{m}$ thick parylene capping layer was deposited, and then patterned using an oxygen plasma with an AZ nLOF 2035 photoresist mask. The etch continued through the first parylene layer in a self-aligned process, which was designed to continue to coat the PVAcTW sidewalls. The photoresist was removed by quickly spraying with acetone and isopropanol, and then drying using an air gun. Finally, the devices were released from the wafer using an aluminum dissolution process [28], as shown in figure 3 .

\section{Mechanical characterization}

A custom-built microtensile tester, shown schematically in figure 4 (top), was constructed specifically for mechanical characterization of the PVAc-TW microstructure in dry, wet, and transition states. The main components of this test setup are a linear piezomotor (MicroMo) capable of displacement in $30 \mathrm{~nm}$ increments, an indicator (Mitutoyo 543-562A) with a $0.5 \mu \mathrm{m}$ resolution, and a load cell (MDB-25, Transducer Techniques) with a resolution of $49 \mu \mathrm{N}$. To prevent samples in the wet state from drying, the tensile tester was designed to incorporate a reservoir to immerse the sample in a fluid (i.e. water) throughout the testing process.

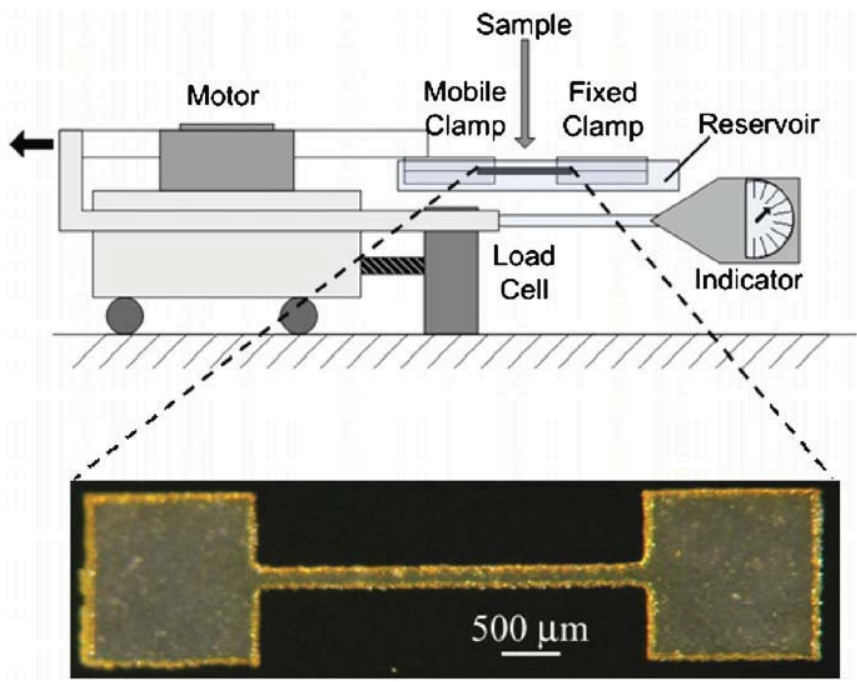

Figure 4. Top: tensile test setup for measuring Young's modulus of widely varying stiffness of micron-scale beams; bottom: example of the laser-cut tensile structure.

Dogbone-shaped samples (figure 4, bottom) were gripped on either end-pad between two acrylic clamps, one fixed and one attached to the piezomotor drive rod. The motor applied a tensile load to the sample, while the indicator measured the pull displacement, and the load cell measured the pull force. All instrumentation was computer-controlled with data acquisition software (DAQFactory, Azeotech).

Displacement and force data can be converted to strain and stress, respectively, by dividing displacement by the initial length, and dividing force by the initial cross-sectional area of the sample. Young's modulus of the sample is defined as the slope of the linear portion of the stress-strain curve. To ensure that the microtensile tester was operating properly, Young's modulus of laser-patterned, $75 \mu \mathrm{m}$ thick Kapton samples was measured, and compared with literature values.

The microtensile tester was operated in two different modes to evaluate the mechanical behavior of micromachined PVAc-TW test structures. In tension mode, the samples were pulled in either the dry or saturated wet state until either the sample broke or the maximum range of the tensile tester was reached. In dynamic mode, the samples were cycled between loading (stretching) and unloading (returning to the initial state) within the linear elastic region, to determine the sample stiffness as a function of time. The former allows for determining parameters such as tensile strength and strainto-break, while the latter enables multiple measurements of Young's modulus in dry, wet, and transitional states. Each dogbone-shaped test structure was $3000 \mu \mathrm{m}$ long, $180 \mu \mathrm{m}$ wide and $50 \mu \mathrm{m}$ thick, with grip pads on either end, $1500 \mu \mathrm{m} \times 1500 \mu \mathrm{m}$. Wet samples were soaked in deionized (DI) water in the reservoir for $1 \mathrm{~h}$ prior to testing.

Results of tension-mode testing are shown in figure 5. Young's modulus of dry samples $(n=3)$ was measured to be $3420 \pm 98 \mathrm{MPa}$, and the dry samples were observed to be relatively brittle with a tensile strength of $56.7 \pm 5.9 \mathrm{MPa}$ at a strain-to-break of $2.9 \% \pm 0.4 \%$. For comparison, polyimide (Kapton HN) has a Young's modulus of $2500 \mathrm{MPa}$, a tensile 


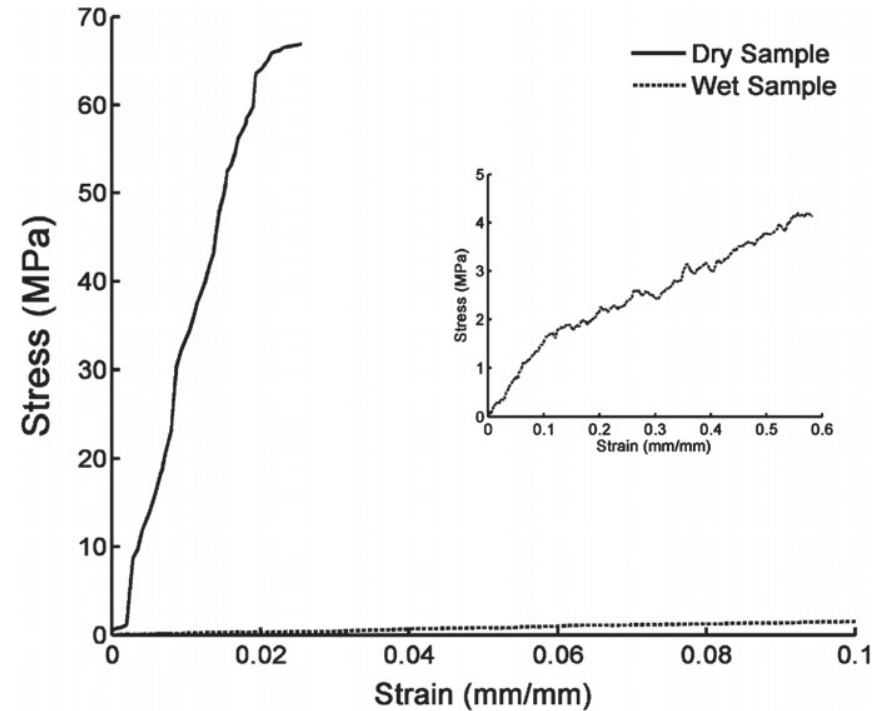

Figure 5. Stress-strain plot of PVAc-TW microsamples demonstrating a dramatic modulus difference in dry and wet samples. The inset focuses on the stress-strain behavior of the wet sample at room temperature, which even at very large strains was deformed with relatively small stress.

strength of $231 \mathrm{MPa}$, and a strain-to-break of $72 \%$ [29]. As polyimide is relatively moisture resistant compared to PVAcTW, immersion in DI water does not appreciably alter the mechanical properties of polyimide, which was demonstrated with the microtensile tester. However, Young's modulus of PVAc-TW was drastically reduced to $22 \pm 7 \mathrm{MPa}$ upon immersion in DI water. Further, the strain-to-break was dramatically increased to $>500 \%$. The tensile toughness, a measure of the energy required to fracture a sample, as delineated by the area under the stress-strain curve, was also increased from $0.97 \mathrm{MPa}$ in the dry state to $>1.46 \mathrm{MPa}$ in the wet state. Thus, after absorbing moisture, PVAc-TW requires significantly more energy to break a sample than it does in the dry state.

It was previously demonstrated that the modulus in dehydrated bulk PVAc-TW specimens driven to full saturation was completely restored upon drying [7]. This behavior was assessed for the micromachined structures by first soaking the laser-cut tensile structures in DI water for $2 \mathrm{~h}$, and then drying the samples in ambient conditions for $24 \mathrm{~h}$, followed by tensile testing. After $24 \mathrm{~h}$, the samples recovered much of their initial stiffness, with a measured Young's modulus of $2900 \mathrm{MPa}$. The full stiffness was recovered by placing ambient-dried samples under vacuum for $2 \mathrm{~h}$, as shown in figure 6 . These results demonstrate that the stimulus-responsive, reversible behavior of PVAc-TW translates to the micron scale, and is not influenced by the laser processing.

The microtensile tester was also operated in dynamic mode to measure the rate of modulus change upon exposure to hydrogen-bond forming liquids like DI water. Dry samples were loaded into the tester, and the empty reservoir was set underneath the sample. The samples were then strained at a rate of $90 \mu \mathrm{m} \mathrm{min}^{-1}$ until a strain of $0.7 \%$ was reached, at which point the motor reversed direction to return to its

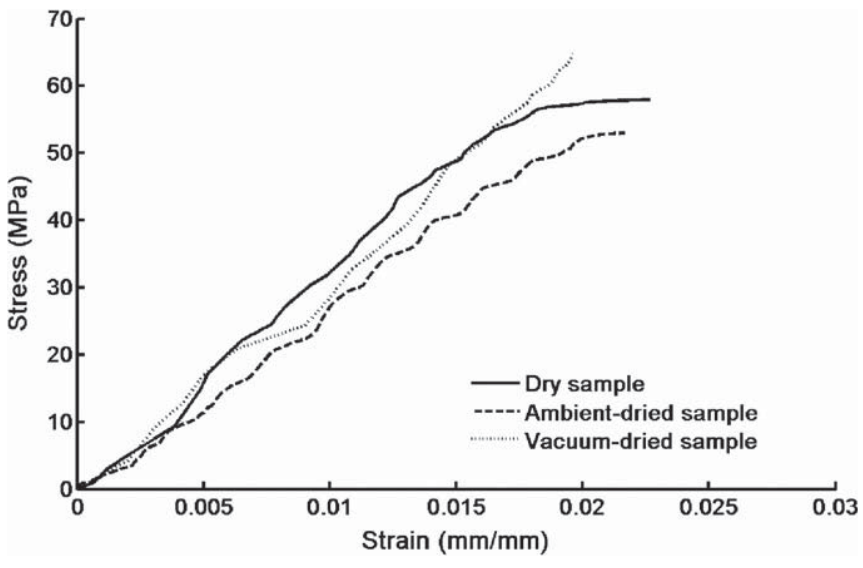

Figure 6. Stress-strain plots of PVAc-TW microsamples, showing the reversibility of switchable stiffness.

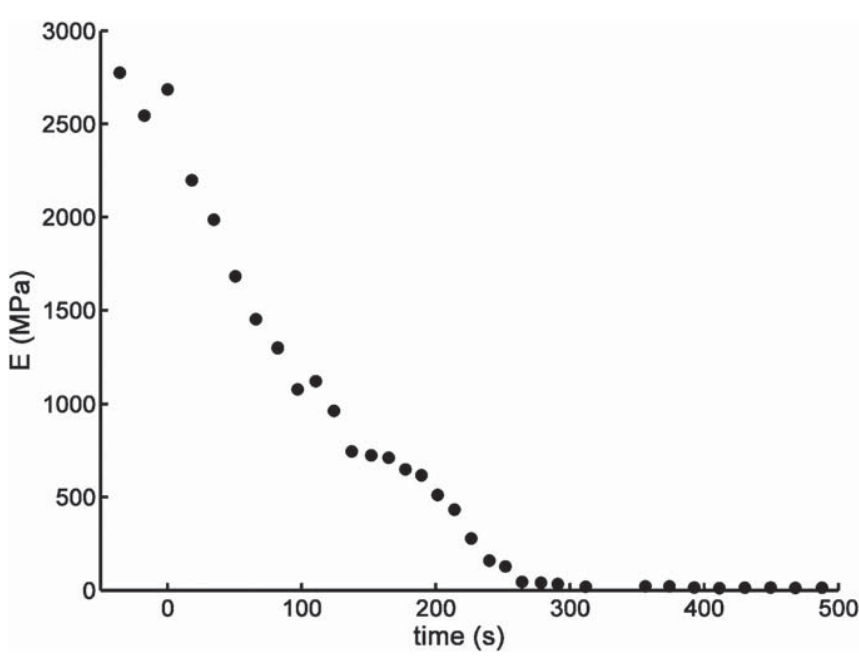

Figure 7. Young's modulus of PVAc-TW versus time exposed to DI water at $20^{\circ} \mathrm{C}$.

initial position. This cycle was repeated throughout the test. After approximately five cycles, the reservoir was filled with DI water, completely immersing the sample. Subsequent loading-unloading cycles allowed for Young's modulus measurement after a known time exposed to DI water.

Young's modulus as a function of time exposed to DI water is shown in figure 7 for a representative sample with dimensions $3000 \mu \mathrm{m} \times 300 \mu \mathrm{m} \times 100 \mu \mathrm{m}$. DI water was introduced at $t=0 \mathrm{~s}$. The stiffness began to decrease nearly instantaneously, with an initial linear drop of $13.3 \mathrm{MPa} \mathrm{s}^{-1}$ for the first $120 \mathrm{~s}$, and the complete change requiring about $300 \mathrm{~s}$. This is a much faster rate of change than was measured for bulk samples, which required 15 min to complete the switch at $37^{\circ} \mathrm{C}$ [7], presumably due to the much larger surface areato-volume ratio of the micromachined samples.

The findings that the laser-micromachined PVAc-TW samples display a change in Young's modulus that is comparable to the razor-blade-cut bulk samples, with a switching speed higher than that of bulk samples, are significant because they show that the micromachining techniques do not significantly alter the stimuli-responsive behavior of the nanocomposite. As laser micromachining 
with a $\mathrm{CO}_{2}$ laser is inherently a thermally destructive process, and because it is known that the PVAc-TW films are sensitive to high temperatures, it was possible that the noncovalent hydrogen-bond interactions between the cellulose nanofibers would be damaged by laser processing. While these mechanical tests do not specifically assess the contribution of the laser-micromachined sidewalls on the mechanical behavior of the PVAc-TW, it is clear that at dimensions relevant to intracortical probes, the dynamic behavior remained intact.

Based on the measured Young's modulus of dehydrated PVAc-TW samples and a typical force required to penetrate the pia mater of the rat, the probe dimensions required to penetrate the rat pia can be calculated. The buckle strength of the probe must be greater than the penetration force, and can be calculated using Euler's buckling formula (equation (1)) if the probe tip does not laterally deflect:

$$
P_{\text {buckle }}=\frac{\pi^{2} E w t^{3}}{6 l^{2}},
$$

where $E, w, t$, and $l$ are Young's modulus, width, thickness and length of the probe, respectively. Najafi et al reported a penetration stress through the rat pia of $1.2 \times 10^{7}$ dynes $\mathrm{cm}^{-2}$ for a $\mathrm{Si}$ probe that was $40 \mu \mathrm{m}$ in thickness and $80 \mu \mathrm{m}$ in width, corresponding to a penetration force of $3.84 \mathrm{mN}$ [12]. Based on these data, for a dry PVAc-TW probe with a Young's modulus of $3.4 \mathrm{GPa}$, a length of $2 \mathrm{~mm}$, and a thickness of $40 \mu \mathrm{m}$, the width would need to be greater than $43 \mu \mathrm{m}$ to penetrate the rat pia without buckling. Increasing the probe length to $3 \mathrm{~mm}$ requires that the width be increased to greater than $97 \mu \mathrm{m}$. These dimensions are well within the range of conventional microfabricated cortical probes. At this point, solution-cast and compressed PVAc-TW films are a minimum of 50 to $60 \mu \mathrm{m}$ in thickness. Since the buckle strength is proportional to the cube of the beam thickness, a small increase in probe thickness provides a considerable improvement to the buckling strength, and the probe width may be reduced. Thus, PVAc-TW probes described in this paper are expected to penetrate the rat pia, but this will be a focus of further investigation. Penetration of the rat dura requires considerably more force [12], but based on the penetration stress of $3.7 \times$ $10^{8}$ dynes $\mathrm{cm}^{-2}$ for a $40 \mu \mathrm{m}$ thick and $80 \mu \mathrm{m}$ wide Si probe, the rat dura may be penetrated with a PVAc-TW-based probe by increasing the cross-sectional dimensions to $100 \mu \mathrm{m} \times$ $100 \mu \mathrm{m}$.

\section{Characterization of multilayered structures}

\subsection{Mechanical testing: water-saturated PVAc-TW samples with parylene coating}

Tensile testing of a water-saturated $100 \mu \mathrm{m}$ thick PVAc-TW sample coated on one surface with a $1 \mu \mathrm{m}$ thick parylene film was performed to show that the sample still exhibited a reduced Young's modulus upon exposure to DI water. The results, shown in figure 8, yielded a Young's modulus of $\sim 25 \mathrm{MPa}$, which is not significantly higher than the Young's modulus of the bare, water-saturated sample (22 MPa). Based on a simple law of mixtures estimate, and on the through-thickness swelling of PVAc-TW at room temperature, it was expected

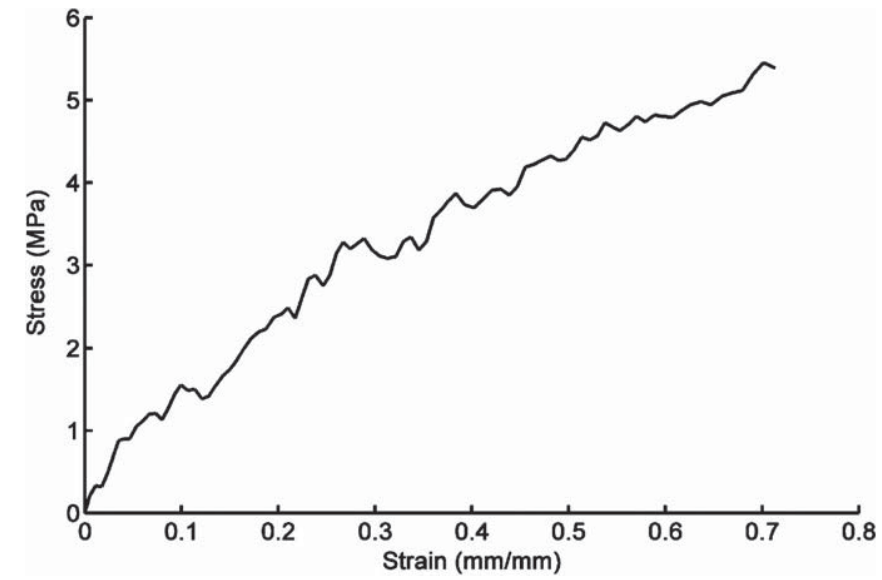

Figure 8. Stress-strain plot of a water-swollen PVAc-TW sample with a $1 \mu \mathrm{m}$ thick parylene $\mathrm{C}$ coating.

that the Young's modulus of the multilayered structure would be $\sim 42 \mathrm{MPa}$. The fortuitous difference between expected and measured is likely due to the fact that the law of mixtures applies to a homogeneous composite and not the parylenecoated PVAc-TW. Further, it can be expected that while the PVAc-TW is able to stretch significantly in the wet state, the parylene coating will likely fracture at high strains.

\subsection{Leakage current through PVAc-TW and parylene-coated PVAc-TW}

To compare the insulating behavior of PVAc-TW with and without a parylene coating, interdigitated electrodes were used to measure the effect of moisture absorption on the leakage current. A $25 \mu \mathrm{m}$ thick parylene film was deposited on a Si wafer for electrical insulation, followed by the deposition and lithographic patterning of $30 \mathrm{~nm} \mathrm{Ti} / 250 \mathrm{~nm} \mathrm{Al}$ electrodes. Each electrode had 25 digits that were $1 \mathrm{~cm}$ long $\times 100 \mu \mathrm{m}$ wide, with $50 \mu \mathrm{m}$ spacing between adjacent digits. In one set of electrodes, PVAc-TW served as a capping layer; in a second set, a $1 \mu \mathrm{m}$ thick parylene $\mathrm{C}$ film was deposited onto the electrodes prior to adding the PVAc-TW capping layer. Thus, the insulating layer of one device was PVAc-TW, and for the other was parylene C/PVAc-TW.

Custom-made reservoirs were attached, using doublesided Kapton tape, to each set of electrodes to allow for selective exposure of the capping layer to phosphate buffered saline (PBS), which was used to simulate the electrolytic fluid in the biological environment. The leakage current between the electrodes was measured versus time exposed to PBS while a dc potential of $3 \mathrm{~V}$ was applied between the electrodes. The leakage current, shown in figure 9 , for the capping layer comprising only PVAc-TW was constant at $\sim 7.5 \mathrm{nA}$ for the first $20 \mathrm{~min}$ of exposure to PBS. After this initial period, the leakage current steadily increased for $4.5 \mathrm{~h}$, at which point a maximum current of $208 \mathrm{nA}$ was measured. Subsequently, the leakage current decreased, presumably due to anodic dissolution of the Al. In sharp contrast, the leakage current for the capping layer comprising a parylene insulating/moisture barrier layer was stable at $\sim 3.8 \mathrm{nA}$ for 


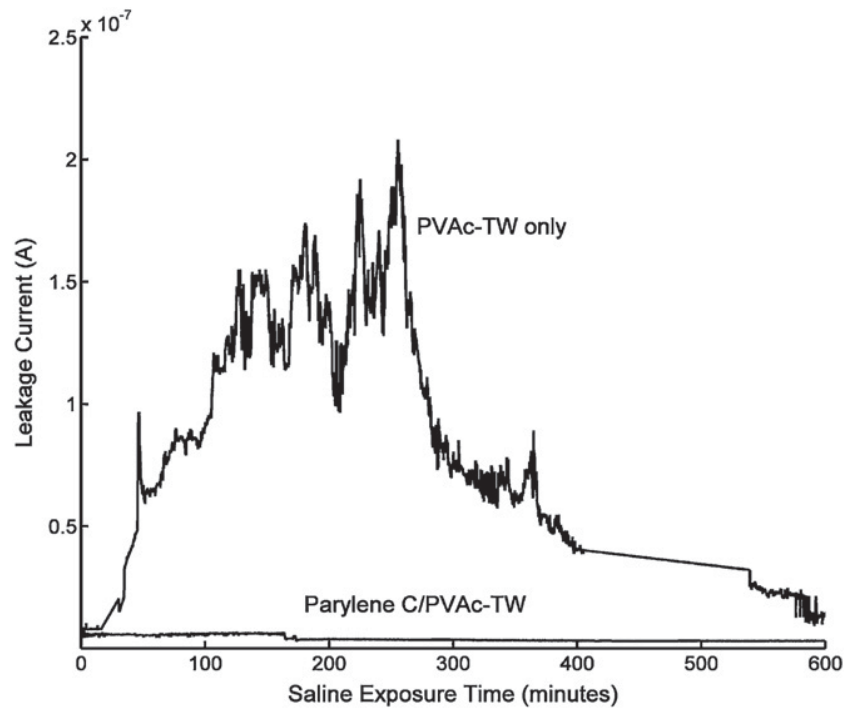

Figure 9. Leakage current between interdigitated electrodes as a function of time exposed to PBS through PVAc-TW or parylene.

more than $24 \mathrm{~h}$. While water uptake of non-passivated PVAcTW-based devices causes a significant leakage current, this problem can be simply circumvented by application of a thin parylene insulating layer.

One potential concerns that one might have about utilizing PVAc-TW as a substrate for metallized medical implants is the potential for metal corrosion and electrical crosstalk between adjacent channels due to electrolyte absorption. The dynamic response is completely dependent on the PVAc-TW being able to absorb and expel liquids, and thus at least one surface of the PVAc-TW-based device must be exposed to the environment. Gratifyingly, the above leakage current data suggest that a $1 \mu \mathrm{m}$ thick parylene $\mathrm{C}$ barrier layer between the water-responsive substrate and the electrodes is sufficient to electrically insulate these two crucial device elements and guarantee their respective functionality, at least in the timeframe of the experiments conducted here.

\subsection{Adhesion and swelling}

Adhesion of the parylene and metal films to PVAc-TW was assessed using both a Scotch tape test and a saline soak test. The tape test was performed on dry samples by pressing a piece of pressure-sensitive tape onto the parylene-coated side of metallized PVAc-TW structures, and then pulling up on the tape. No delamination of the parylene or metal films from the PVAc-TW substrate was observed, indicating that the adhesion of these films is strong for the as-fabricated structures. Long-term exposure to the harsh biological environment was simulated by soaking three PVAc-TW probes in PBS at $37{ }^{\circ} \mathrm{C}$ for 60 days. Again, no delamination or degradation was observed after the soak test, indicating sufficient adhesion for at least short-term chronic studies.

Upon soaking in PBS at elevated temperatures, it was noted that the multilayer structures did not curl upon PBS absorption, in spite of the relatively large stiffness difference of the different materials after aqueous swelling of the nanocomposite. The PVAc-TW devices were designed to

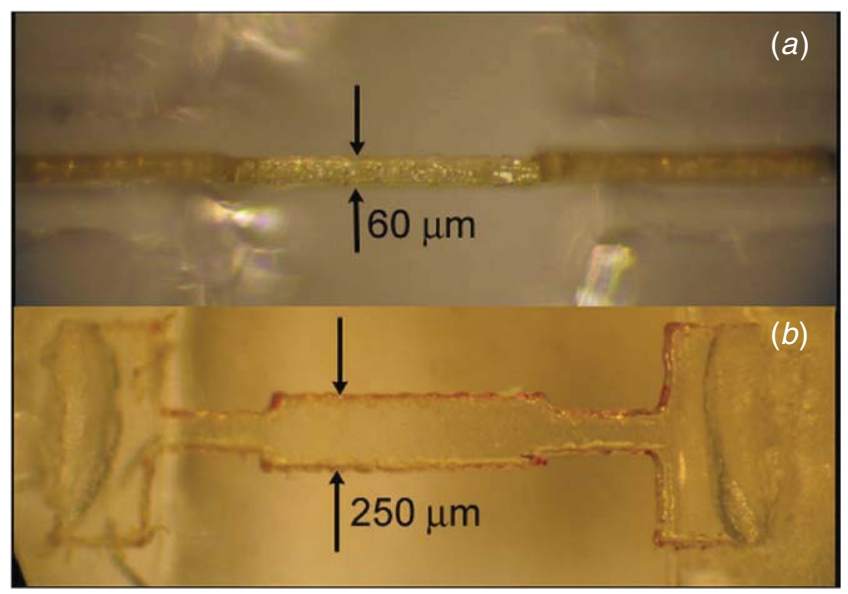

Figure 10. (a) PVAc-TW thickness measurement; (b) PVAc-TW width measurement.

incorporate moisture-resistant, non-swelling thin films onto the surface of a comparatively thicker PVAc-TW substrate. Thus, it would be expected that a large amount of lateral swelling would cause curling of the device, with the parylene and metal films under tensile stress. To investigate this phenomenon, a study was completed to determine how the moisture absorption was distributed throughout the sample volume. Swelling measurements were performed on micromachined PVAc-TW samples to determine the increase in the lateral and thickness dimensions upon exposure to DI water. Acrylic jigs were assembled to hold the samples and allow for sustained immersion in water. One set of test structures was used to measure the dimensional increase through the thickness of the film (figure $10(a)$ ), and a second set was used to measure the increase across the plane of the film (figure 10(b)). The critical dimension (thickness or width) of the samples was measured under a microscope both before and after exposure to DI water at either room temperature $\left(20^{\circ} \mathrm{C}\right)$ or body temperature $\left(37^{\circ} \mathrm{C}\right)$ for $1 \mathrm{~h}$.

A comparison of the measurements is shown in figure 11. At room temperature, the lateral increase was measured to be $\sim 3 \%$, and the increase through the plane of the film was three times that at $\sim 24 \%$, indicating anisotropic swelling with a through-plane to across-plane ratio of $\sim 8$. This swelling behavior may be the result of the compression step in film synthesis, in which the compressive force is applied in the through-plane dimension. The anisotropic swelling was even more pronounced at $37{ }^{\circ} \mathrm{C}$, with a through-plane to across-plane ratio of 12 . These results are consistent with the volume swelling, by weight, reported for the bulk material at $37{ }^{\circ} \mathrm{C}$ of $\sim 70 \%$. The anisotropic swelling is advantageous for the proposed device architecture and fabrication sequence. As much of the swelling is through the thickness of a planar device, through-plane distortion is expected to be reduced or eliminated.

\subsection{Electrochemical impedance spectroscopy}

Electrochemical impedance spectroscopy of a PVAc-TWbased probe (shown in figure 3 , bottom) versus a Pt reference electrode was performed in PBS. Contact to the probe was 


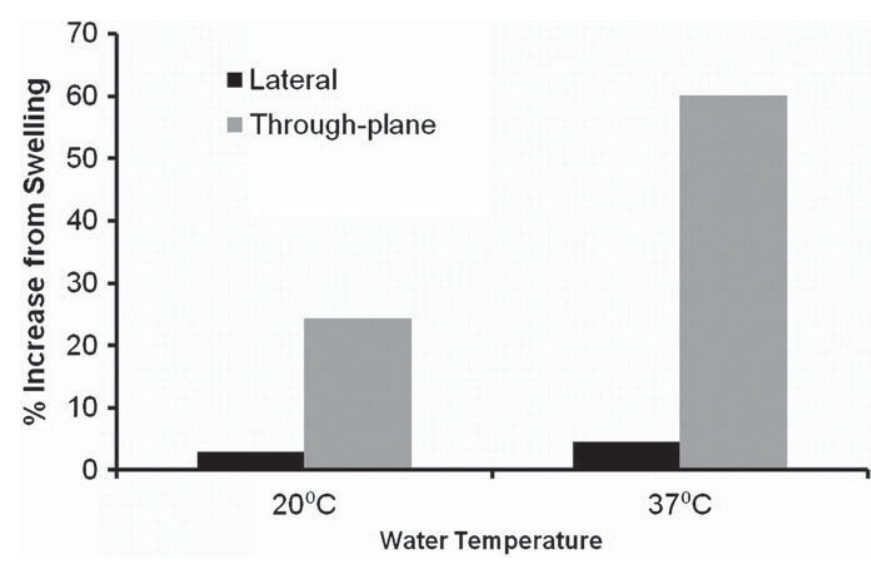

Figure 11. Anisotropic swelling of PVAc-TW in 20 and $37^{\circ} \mathrm{C}$ deionized water.
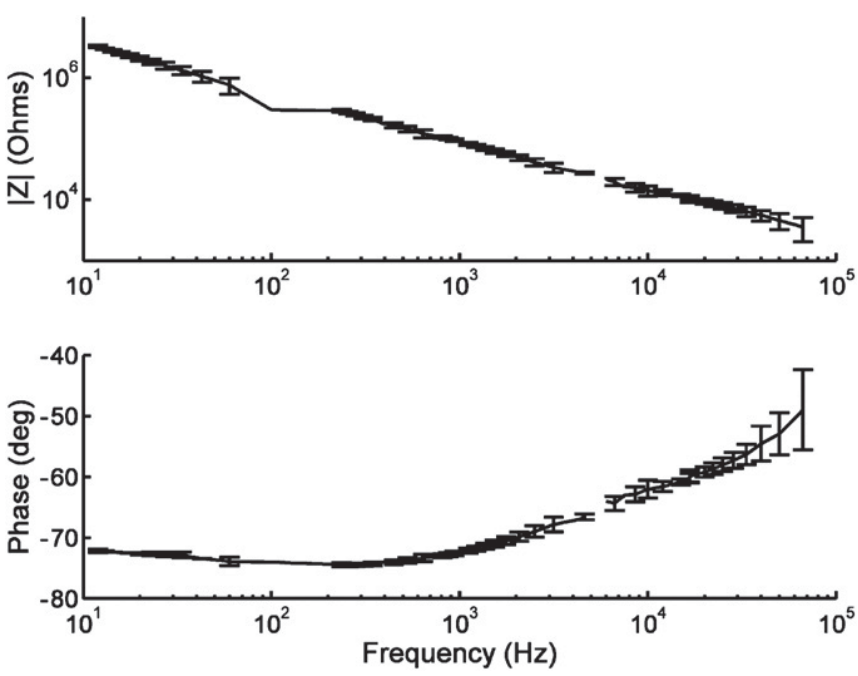

Figure 12. Electrochemical impedance spectra of a PVAc-TW cortical probe with a $6000 \mu \mathrm{m}^{2}$ Au electrode.

made with a wire attached to the exposed contact pad using conductive epoxy. An LCR meter was used to make the measurements, scanning through frequencies from $10 \mathrm{~Hz}$ to $200 \mathrm{kHz}$. The results are shown in figure 12 . At $1 \mathrm{kHz}$, the impedance was measured to be $95.0 \pm 4.8 \mathrm{k} \Omega$. The electrode-electrolyte interface for an Au electrode in PBS can be modeled as an electrode resistance, $R_{e}$, in parallel with an electrode capacitance, $C_{e}$, these values being highly dependent upon electrode area with $R_{e}$ estimated as $6.5 \mathrm{M} \Omega \mathrm{cm}^{2}$ and $C_{e}$ estimated as $20 \mathrm{mF} \mathrm{cm}^{-2}$ for an Au electrode [30]. For an Au electrode with an area of $6000 \mu \mathrm{m}^{2}$, the magnitude of the impedance at a frequency of $1 \mathrm{kHz}$ was estimated to be $130 \mathrm{k} \Omega$, which is highly comparable to the measured value. This suggests that not only has a $30 \mu \mathrm{m}$ wide trace successfully connected the electrode to the contact pad, and was continuous despite the rough PVAc-TW surface, but also that the PVAcTW substrate did not affect the quality of the Au film or the behavior of the electrode-electrolyte interface.

\section{Conclusions}

A bio-inspired, mechanically dynamic, chemo-responsive polymer nanocomposite with a poly(vinyl acetate) matrix has been utilized as a substrate material in the fabrication of variable-modulus microstructures. Laser micromachining was identified as a method for producing PVAc-TW microstructures, and it was shown that these microstructures display a reversible change in Young's modulus upon exposure to DI water that is comparable to measurements on the bulk material. In contrast to the bulk material, PVAc-TW microstructures switch from stiff to flexible in $\sim 5 \mathrm{~min}$ at room temperature. It was found that PVAc-TW displays anisotropic swelling behavior, with 8-12 times more swelling through the film thickness than across the film. Utilizing a thin parylene film as a thin film barrier protecting the chemically sensitive PVAc from photoresist solvent and developer solutions, metal features could be successfully fabricated using conventional photolithography. These multilayer devices demonstrated good adhesion, and had an impedance comparable to other $\mathrm{Au}$ electrodes. Because the multilayer fabrication process described here does not require the polymer to be exposed directly to acids, bases, or organic solvents, this process can be extended for any moisture-absorbent polymer.

\section{Acknowledgments}

This work was funded by NSF grants ECS-0621984 and CBET-0828155, NIH contract R21-NS053798, and Advanced Platform Technology Center of Excellence of The Department of Veteran's Affairs. CW is grateful for the funding from SNF (406240_126046/1) under the NFP 62 program.

\section{References}

[1] Schmaljohann D 2006 Thermo- and pH-responsive polymers in drug delivery Adv. Drug Deliv. Rev. 58 1655-70

[2] Bassetti M J, Chatterjee A N, Aluru N R and Beebe D J 2005 Development and modeling of electrically triggered hydrogels for microfluidic applications Microelectromech. Syst. J. 14 1198-207

[3] Motokawa T 1994 Effects of ionic environment on viscosity of Triton-extracted catch connective tissue of a sea cucumber body wall Comp. Biochem. Physiol. B 109 613-22

[4] Trotter J A et al 2000 Towards a fibrous composite with dynamically controlled stiffness: lessons from echinoderms Biochem. Soc. Trans. 28 357-62

[5] Thurmond F and Trotter J 1996 Morphology and biomechanics of the microfibrillar network of sea cucumber dermis J. Exp. Biol. 199 1817-28

[6] Wilkie I C 2002 Is muscle involved in the mechanical adaptability of echinoderm mutable collagenous tissue? J. Exp. Biol. 205 159-65

[7] Capadona J R, Shanmuganathan K, Tyler D J, Rowan S J and Weder C 2008 Stimuli-responsive polymer nanocomposites inspired by the sea cucumber dermis Science 319 1370-4

[8] Shanmuganathan K, Capadona J R, Rowan S J and Weder C 2009 Stimuli-responsive mechanically adaptive polymer nanocomposites ACS Appl. Mater. Interfaces 2 165-74

[9] Šturcová A, Davies G R and Eichhorn S J 2005 Elastic modulus and stress-transfer properties of tunicate cellulose whiskers Biomacromolecules 61055

[10] Shanmuganathan K, Capadona J R, Rowan S J and Weder C 2010 Biomimetic mechanically adaptive nanocomposites Prog. Polym. Sci. 35 212-22 
[11] Hess A, Dunning J, Harris J, Capadona J R, Shanmuganathan K, Rowan S J, Weder C, Tyler D J and Zorman C A 2009 A bio-inspired, chemo-responsive polymer nanocomposite for mechanically dynamic microsystems Proc. Solid-State Sensors, Actuators and Microsystems Conf. (Transducers) (June 2009) pp 21-5

[12] Najafi K and Hetke J F 1990 Strength characterization of silicon microprobes in neurophysiological tissues IEEE Trans. Biomed. Eng. 37474

[13] Lee K-K, He J, Singh A, Massia S, Ehteshami G, Kim B and Raupp G 2004 Polyimide-based intracortical neural implant with improved structural stiffness J. Micromech. Microeng. 1432

[14] Subbaroyan J, Martin D C and Kipke D R 2005 A finite-element model of the mechanical effects of implantable microelectrodes in the cerebral cortex J. Neural Eng. 2103

[15] Lee H, Bellamkonda R V, Sun W and Levenston M E 2005 Biomechanical analysis of silicon microelectrode-induced strain in the brain J. Neural Eng. 281

[16] Williams J C, Hippensteel J A, Dilgen J, Shain W and Kipke D R 2007 Complex impedance spectroscopy for monitoring tissue responses to inserted neural implants J. Neural Eng. 4410

[17] Polikov V S, Tresco P A and Reichert W M 2005 Response of brain tissue to chronically implanted neural electrodes J. Neurosci. Methods 148 1-18

[18] Szarowski D H, Andersen M D, Retterer S, Spence A J, Isaacson M, Craighead H G, Turner J N and Shain W 2003 Brain responses to micro-machined silicon devices Brain Res. $98323-35$

[19] Rousche P J, Pellinen D S, Pivin D P Jr, Williams J C, Vetter R J and Kipke D R 2001 Flexible polyimide-based intracortical electrode arrays with bioactive capability IEEE Trans. Biomed. Eng. 48 361-71

[20] Metz S, Bertsch A, Bertrand D and Renaud P 2004 Flexible polyimide probes with microelectrodes and embedded microfluidic channels for simultaneous drug delivery and multi-channel monitoring of bioelectric activity Biosens. Bioelectron. 19 1309-18

[21] Takeuchi S, Ziegler D, Yoshida Y, Mabuchi K and Suzuki T 2005 Parylene flexible neural probes integrated with microfluidic channels Lab Chip 5 519-23

[22] Soza G, Grossa R, Nimsky C, Greiner G and Hastreiter P 2004 Estimating Mechanical Brain Tissue Properties with Simulation and Registration (Lecture Notes in Computer Science) (Berlin: Springer) pp 276-83

[23] Soza G, Grosso R, Nimsky C, Hastreiter P, Fahlbusch R and Greiner G 2005 Determination of the elasticity parameters of brain tissue with combined simulation and registration Int. J. Med. Robot. Comput. Assist. Surg. 1 87-95

[24] Khoshgoftar M, Najarian S, Farmanzad F, Vihidi B and Ghomshe F T 2007 A biomechanical composite model to determine effective elastic moduli of the CNS gray matter Am. J. Appl. Sci. 4918

[25] Rusli R, Shanmuganathan K, Rowan S J, Weder C and Eichhorn S J 2010 Stress-transfer in anisotropic and environmentally adaptive cellulose whisker nanocomposites Biomacromolecules 11 762-8

[26] Shanmuganathan K, Capadona J R, Rowan S J and Weder C 2010 Bio-inspired mechanically-adaptive nanocomposites derived from cotton cellulose whiskers J. Mater. Chem. 20180

[27] V\&P Scientific, Inc. 2010 Parylene properties and characteristics http://www.vp-scientific.com/parylene_properties.htm

[28] Metz S, Holzer R and Renaud P 2001 Polyimide-based microfluidic devices Lab Chip $129-34$

[29] DuPont 2006 DuPont ${ }^{\mathrm{TM}}$ Kapton ${ }^{\circledR} \mathrm{HN}$ polyimide film http://www2.dupont.com/Kapton/en_US/assets/downloads/ pdf/HN_datasheet.pdf

[30] Wise K D and Angell J B 1975 A low-capacitance multielectrode probe for use in extracellular neurophysiology IEEE Trans. Biomed. Eng. 22 212-9 